\title{
Retrograde capabilities of adeno-associated virus vectors in the central nervous system
}

\author{
MAgdalena M. SuRdyKa *, MACIEJ Figiel \\ Institute of Bioorganic Chemistry, Polish Academy of Sciences, Poznań, Poland
}

\begin{abstract}
Adeno-associated virus (AAV) vectors delivered at the axonal terminals can be retrogradely transported toward neuronal cell bodies throughout the axons. This retrograde phenomenon can serve as a powerful tool for experiments and gene therapy using AAVs. The advantages of using AAV vectors delivered retrogradely are greater cellular specificity, high transduction efficiency, increased safety, and absence of cytotoxicity. The numerous axonal projections in the nervous system provide a neuronal network for the convenient and widespread distribution of viral vectors between adjacent brain structures and over long distances. The retrograde efficiency of AAVs in the neurons of the central nervous system (CNS) depends on AAV serotype, the region of injection, and the type of neurons. In this review, we describe AAV serotypes and their retrograde transport properties after injection and discuss brain structures or types of cells that are targeted for retrograde transport. In particular, AAV serotypes $2,5,8,9$, rh10, and PHP.eB are extensively reviewed as they demonstrate retrograde transport potential suitable for use in gene therapy applications.
\end{abstract}

Key words: adeno-associated virus, serotype, retrograde transport, central nervous system

\section{Introduction}

Axonal transport is a critical physiological process involving the transfer of material between the cell body and the axonal terminal of neurons. Materials delivered for the axons are transported in the anterograde direction toward the axonal terminal. Materials returning to the cell body are transported retrogradely toward the cell body (Fig. 1; Tervo et al., 2016). The retrograde transport phenomenon in neurons is the basis for delivering therapeutic molecules by viral vectors. Adenoassociated virus (AAV) vectors are a diverse group of tools for gene therapy that have shown significant evidence of safety and transduction efficacy in several species. They are the most frequently used gene therapy vectors for the central nervous system (CNS) as they are nonpathogenic and can transduce dividing and quiescent cells in vivo (Choudhury et al., 2017; Hadaczek et al., 2010). The important characteristics of the AAV viral vectors are their negligible immunogenicity and absence of cytotoxicity.
The AAV-based expression of therapeutic genes and molecules can be sustained for several months in some cells (Nathwani et al., 2011). The most frequently studied serotypes of AAV vectors are $1,2,5,8,9$, recombinant human (rh)10, and PHP.eB, which additionally demonstrate abilities to be transported along the neuronal processes in both anterograde and retrograde manner. Retrograde transport of AAV vectors is an interesting phenomenon because of the numerous axonal projections in the nervous system. Precise vector delivery to the axonal terminals allows their transport within adjacent brain structures and over long distances. Therefore, there is ongoing research to understand the retrograde properties of strains of other viruses, including adenoviruses, which can infect neurons when administered directly to the nervous system (Cearley and Wolfe, 2006; Dayton et al., 2018; Klein et al., 2008; Wang and Zhang, 2021). Developing a nontoxic, easy-to-produce viral vector that allows flexible packaging of different transgenes, is highly internalized and retrogradely trans-

\footnotetext{
* Corresponding author: Institute of Bioorganic Chemistry, Polish Academy of Sciences, Poznań, Poland; e-mail: msurdyka@ibch.poznan.pl
} 
ported across axons, and supports the long-term, highlevel expression of cargo has been a research goal of many scientific groups for the last two decades. The high therapeutic potential due to the retrograde properties of AAV makes it reasonable to expand our knowledge in this field. Therefore, we reviewed the literature on AAV considering three factors: serotype, region of injection, and region/cell type in which retrograde transport was targeted (Table 1).

\section{AAV serotype 2}

The retrograde properties of the AAV2 vector were reported by Kaspar et al. (2002). AAV2 was directly injected into the axon terminal fields in the rat hippocampus and striatum. This placement of AAV2 in the vicinity of the axons resulted in viral internalization, retrograde transport, and transgene expression in specific projection neurons in the entorhinal cortex and substantia nigra. Therefore, the transgene expression by AAV2 can be located remotely from the injection site of the AAV. In another study, Passini et al. (2005) injected AAV2 into the hippocampus of a mouse model of Niemann-Pick disease. This injection of the vector resulted in the transduction of all primary cell layers of the ipsilateral hippocampus. In addition, the entorhinal cortex, medial septum, and contralateral hippocampus were also transduced, probably due to the retrograde axonal transport of AAV2 particles. Recently, a new AAV2-retro serotype has been generated with enhanced retrograde capabilities as compared to AAV2. The AAV2-retro serotype was used to test the retrograde transport pathway between the pontine medial parabrachial nucleus (MPB) and the cerebellum lobules. To examine axonal terminals of cerebellar Purkinje cells (PCs) projecting to the $\mathrm{MPB}$, the authors unilaterally injected Fast Blue and the AAV2-retro vector as retrograde markers into the MPB. The PC in lobules VIII-X were retrogradely labeled by Fast Blue and AAV2-retro, but no retrograde-labeled PCs were observed in lobules VI-VII and the cerebellar hemispheres. These results indicate that PCs in lobules VIII-X directly project their axons to the ipsilateral MPB, but not those in lobules VI-VII (Hashimoto et al., 2018). Recently, retrograde properties have also been reported for serotype AAV2/DJ and mouse brain striatal injections, which targeted cells in the substantia nigra (Düring et al., 2020). The CNS retrograde capabilities of AAV2 and other serotypes are summarized in Table 1 (Itoga et al., 2019; Jara et al., 2012; Zheng et al., 2020).

\section{AAV serotype 5}

The striatum and thalamus have multiple axonal projections, resulting in dense interconnections with other brain regions. This neuronal network allows for targeting these regions by AAV and retrograde transport in therapeutic approaches for neurodegenerative diseases. Therefore, to investigate the transduction pattern, AAV5-based vectors were injected into the rat striatum and thalamus (Pietersz et al., 2020) (Table 1). Because the transduced cell bodies appeared relatively far from the AAV5 injection site, the authors suggest that this transduction pattern results from retrograde transport. For instance, after striatal injection, vector expression was observed in the cortex, thalamus, and hippocampus and thus at a remote location from the injection site. Aschauer et al. (2013) examined the transport of rAAV serotypes in the CNS. They searched for the presence of green fluorescent protein (GFP)+ cell bodies located at distances of more than $500 \mu \mathrm{m}$ from the injection site that could not be explained by the simple diffusion of the virus. The authors observed that the injection of rAAV5 into the dentate gyrus of the hippocampus led to the labeling of cell bodies in the ipsilateral lateral entorhinal cortex and to a lesser extent in the contralateral entorhinal cortex in all injected mice. To test whether GFP labeling is due to specific retrograde axonal transport of AAV5, the authors co-injected GFP-encoding rAAV5 with the retrograde tracer Cholera Toxin B (CTB). In all injected mice, GFP expression was almost exclusively observed in cells that were also labeled with CTB. Intriguingly, the rAAV5-labeled cells were most frequent in layer 2 of the ventral part of the lateral entorhinal cortex, potentially labeling a distinct sub-population of projection neurons. The retrograde characteristics of the AAV5 serotype in the CNS are well established and are further indicated in other studies (Haenraets et al., 2017).

\section{AAV serotype 8}

The retrograde properties of AAV8 in the CNS (Table 1) have been described in several research studies (Castle et al., 2014; Klaw et al., 2013; Löw et al., 2013; Parfitt et al., 2017; Pina and Cunningham, 2017; Taymans et al., 2007). The retrograde transport of AAV8 was evaluated between the ventral hippocampus and the lateral septum or the medial prefrontal cortex (Parfitt et al., 2017). The authors observed a retrograde migration of the fluorescent mCherry reporter along the axons 
Table 1. Retrograde transduction characteristics of selected AAV serotypes in the CNS

\begin{tabular}{|c|c|c|c|}
\hline Serotype & Region of injection & Retrograde transport target region & Reference \\
\hline AAV2 & hippocampus, striatum & $\begin{array}{l}\text { neurons in the entorhinal cortex, medial } \\
\text { septum, contralateral hippocampus, } \\
\text { and substantia nigra }\end{array}$ & $\begin{array}{l}\text { Kaspar et al., 2002; Passini } \\
\text { et al., } 2005\end{array}$ \\
\hline AAV2-retro & $\begin{array}{l}\text { cerebellum-medial para- } \\
\text { brachial nucleus }\end{array}$ & cerebellar purkinje cells in lobules VIII-X & Hashimoto et al., 2018 \\
\hline AAV2-retro & olfactory cortex & $\begin{array}{l}\text { bed nucleus of the stria terminalis, anterior } \\
\text { cortical amygdalar area, medial amygdaloid } \\
\text { nucleus, and posteromedial cortical amygda- } \\
\text { loid area }\end{array}$ & Zheng et al., 2020 \\
\hline $\mathrm{AAV} 2 / \mathrm{DJ}$ & striatum, area $\mathrm{X}$ & substantia nigra, ventral tegmental area & Düring et al., 2020 \\
\hline AAV5 & striatum, thalamus & $\begin{array}{l}\text { neurons in the cortex, thalamus, and hippo- } \\
\text { campus }\end{array}$ & Pietersz et al., 2020 \\
\hline AAV5 & hippocampus (dentate gyrus) & ipsilateral lateral entorhinal cortex & Aschauer et al., 2013 \\
\hline AAV8 & ventral hippocampus & lateral septum and medial prefrontal cortex & Parfitt et al., 2017 \\
\hline AAV8 & ventral tegmental area & bed nucleus of the stria terminalis & Pina and Cunningham, 2017 \\
\hline AAV8 & striatum & substantia nigra & Masamizu et al., 2011 \\
\hline rAAV8 & hippocampus & deep cerebellar nuclei & Bohne et al., 2019 \\
\hline AAV9 & striatum & substantia nigra & Masamizu et al., 2011 \\
\hline AAV9 & nucleus accumbens shell & ventral tegmental area & Li et al., 2019 \\
\hline AAV9 & ventral tegmental area & substantia nigra & Cearley and Wolfe, 2007 \\
\hline AAV9 & putamen & $\begin{array}{l}\text { thalamic, cortical neurons, and substantia } \\
\text { nigra }\end{array}$ & Green et al., 2016 \\
\hline rAAV9 & $\begin{array}{l}\text { primary motor cortex, visual } \\
\text { cortex, striatum, and somato- } \\
\text { sensory cortex }\end{array}$ & $\begin{array}{l}\text { thalamus, motor cortex, somatosensory cor- } \\
\text { tex, contralateral primary motor cortex, la- } \\
\text { teral geniculate nucleus, substantia nigra, } \\
\text { and motor cortex }\end{array}$ & Commisso et al., 2018 \\
\hline AAVrh10 & ventral tegmental area & substantia nigra & Cearley and Wolfe, 2007 \\
\hline AAVrh10 & striatum & thalamus, cortex ventral tegmental area & Piguet et al., 2012 \\
\hline PHP.eB & Deep cerebellar nuclei & purkinje cells & (unpublished data) \\
\hline
\end{tabular}

from the injection site to the frontal part of the brain, i.e., the lateral septum and the medial prefrontal cortex. Based on immunohistochemical markers and gene expression, Pina and Cunningham (2017) demonstrated the retrograde properties of the AAV8 vector. The authors stereotactically injected the vector into the ventral tegmental area (VTA) and showed that the reporter delivered along with serotype 8 was localized in the VTAprojecting bed nucleus of the stria terminalis (BNST) neurons. The signal observed in axons and bodies of BNST neurons indicates retrograde transport between the VTA and BNST. The AAVs were also tested to determine their retrograde properties and the remote targets when delivered into the striatum. Transduction efficiency and signal tracing were assessed using the fluorescent marker GFP, which was detected in the substantia nigra following AAV8-eGFP or AAV9-eGFP injection into the striatum. The authors found that the nigrostriatal pathway contributed to the retrograde transport of AAV8 (Masamizu et al., 2011). Serotype rAAV8 was used to assess the retrograde transport pathway between the thalamus and the deep cerebellar nuclei (DCN) (Bohne et al., 2019). The thalamic injections of the rAAV8 serotype demonstrated that the GFP signal moved to the DCN.

\section{A A V serotype 9}

AAV9 also showed retrograde properties (Table 1); however, its transduction efficiency of the DCN was lower than that of AAV8. Furthermore, both AAV8 and 


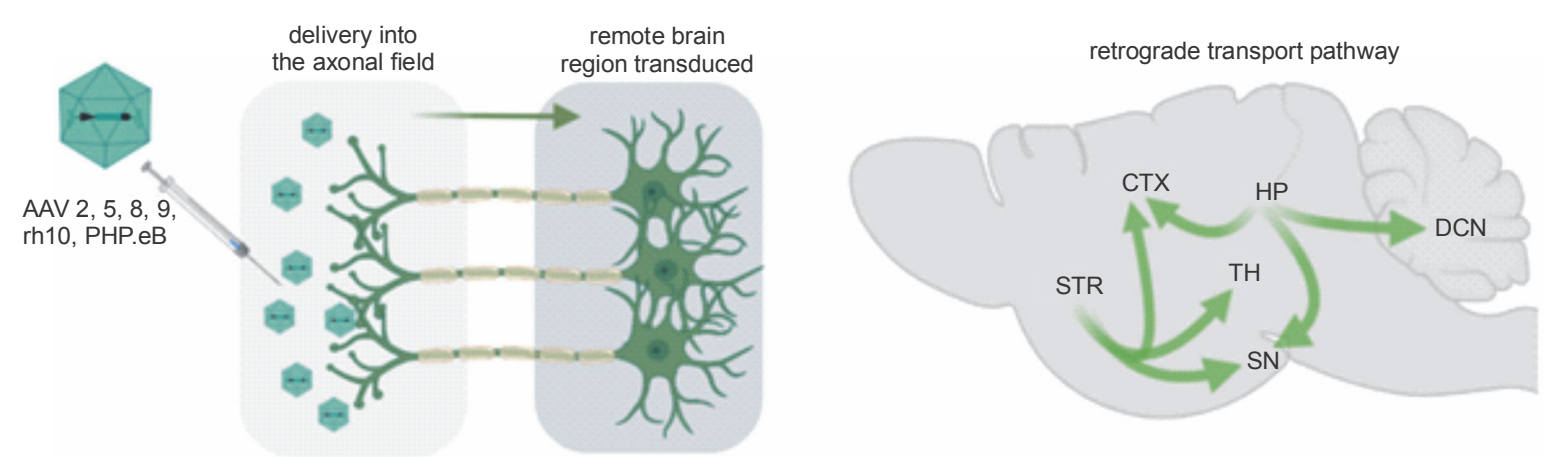

Fig. 1. Retrograde capabilities of AAV vectors. After delivery of the AAV vector into the axonal field in the CNS, axonal retrograde transport occurs, resulting in transgene expression along the axon, neuronal bodies, and dendrites. The advantage of a single injection of AAV into axonal terminals is that it often results in the transduction of even remote brain structures. Natural properties of the brain's neuronal networks allow for simultaneous retrograde delivery of AAV encoding transgenes to many brain regions, which is vital for effective gene therapies. (CTX - cortex, DCN - deep cerebellar nuclei, HP - hippocampus, SN - substantia nigra, STR - striatum, TH - thalamus)

AAV9 serotypes transduced oligodendrocytes along the nigrostriatal pathway (Masamizu et al., 2011). The retrograde transport of the AAV9 vector was also observed between the injection site in the nucleus accumbens shell (AcbSh) and the posterior VTA (Li et al., 2019). Because the AcbSh contains the axonal terminals of VTA neurons (Li et al., 2019), the injection of the AAV9 vector produced an intensive $\mathrm{GFP} / \mathrm{Cre}$ signal along the axons and in the soma of VTA neurons. Therefore, the AAV9 vector seems very well suited for widespread expression throughout the brain; however, the site of delivery into the axonal terminals, which innervate many brain regions, needs to be carefully selected. For instance, the single injection into the striatum or the VTA caused retrograde transport of the vector genome to projection sites in many distal parts of the brain (Cearley and Wolfe, 2007). The authors observed enzyme transport after AAV9 vector injection. They found that its widespread retrograde distribution in the brain resulted in good distribution of the enzyme product and complete correction of the storage lesions throughout the entire brain (Cearley and Wolfe, 2007).

An important study showing translatability to primates demonstrated that 3 weeks after bilateral parenchymal infusion of the AAV9_eGFP virus into each putamen of Rhesus macaques, a green signal was detected in the distal parts of their brain. The GFP expression was detected in thalamic and cortical neurons as well as in dopaminergic neurons projecting from the substantia nigra pars compacta, thus indicating retrograde transport of AAV9 (Green et al., 2016). A new retrograde
AAV9 serotype was used to quantitatively map the projections to the primary motor cortex in pre- and postsymptomatic amyotrophic lateral sclerosis (ALS) mouse, which also demonstrated AAV9 retrograde capabilities. Parenchymal injection of retro-AAV9 increased the number of GFP-positive neurons projecting from the somatosensory cortex to the primary motor cortex at the presymptomatic stages of the disease and increased GFPpositive projections from the auditory cortex and the contralateral motor cortex and thalamus in post-symptomatic mice (Commisso et al., 2018).

\section{AAV serotype rh10}

AAVrh10 is a serotype with prominent retrograde characteristics in the CNS (Cearley and Wolfe, 2007). The axonal transport of the AAVrh10 vector was evaluated after unilateral injection into the striatum, which receives and sends multiple brain signals (Piguet et al., 2012). After a single injection of AAVrh.10 encoding eGFP, the green fluorescent cells were detected in the cellular bodies of neurons in the thalamus, cortex, and VTA, suggesting prominent retrograde transport into distant brain centers.

\section{AAV serotype PHP.eB}

PHP.eB is one of the newer AAV serotypes and a very promising vector for gene therapy of neurodegenerative diseases. Recent studies have demonstrated that the AAV-PHP.eB vector increases the efficiency of wide-scale gene transfer in the CNS as compared to previous serotypes. Additionally, the PHP.eB serotype can 
cross the blood-brain barrier, thereby allowing for minimally invasive treatment (Chatterjee et al., 2021; Dayton et al., 2018; Mathiesen et al., 2020; Xie et al., 2021). The retrograde features of the AAV-PHP.eB remain to be investigated. The studies conducted to date, however, demonstrate widespread transduction of various brain centers by the AAV-PHP.eB serotype, thus indicating its retrograde features.

\section{Conclusion}

The AAV serotypes 2, 5, 8, 9, rh10, and PHP.eB have retrograde properties that can be used in therapeutic applications. For most serotypes analyzed in this review, the hippocampus, cortex, and striatum show tremendous potential for retrograde transport within the CNS. The AAV retrograde approach offers many advantages, including increased precision of brain structure targeting and less invasive brain delivery. Moreover, the delivery approach requires fewer AAV vectors for gene therapy and prevents side effects such as brain inflammation.

This work was supported by the National Centre for Research and Development (grant numbers: ERA-NET-E-RARE-3/III/ TreatPolyQ/08/2018 and ERA-NET-E-RARE-3/III/SCA-CYP/ 09/2018).

\section{References}

Aschauer D.F., Kreuz S., Rumpel S. (2013) Analysis of transduction efficiency, tropism and axonal transport of $A A V$ serotypes 1, 2, 5, 6, 8 and 9 in the mouse brain. PLoS One 8(9): e76310.

Bohne P., Schwarz M.K., Herlitze S., Mark M.D. (2019) In mice, a new projection from the deep cerebellar nuclei to the hippocampus via the ventrolateral and laterodorsal thalamus. Front. Neural Circ. 13: 51.

Castle M.J., Gershenson Z.T., Giles A.R., Holzbaur E.L., Wolfe J.H. (2014) Adeno-associated virus serotypes 1, 8, and 9 share conserved mechanisms for anterograde and retrograde axonal transport. Human Gene Ther. 25(8): 705-720.

Cearley C.N., Wolfe J.H. (2007) A single injection of an adenoassociated virus vector into nuclei with divergent connections results in widespread vector distribution in the brain and global correction of a neurogenetic disease. $\mathrm{J}$. Neurosci. 27(37): 9928-9940.

Mathiesen S.N., Lock J.L., Schoderboeck L., Abraham W.C., Hughes S.M. (2020) CNS transduction benefits of AAVPHP.eB over AAV9 are dependent on administration route and mouse strain. Mol. Ther. Meth. Clin. Develop. 19: 447-458.

Chatterjee D., Marmion D.J., McBride J.L., Manfredsson F.P., Butler D., Messer A., Kordower J.H. (2021) Enhanced
CNS transduction from AAV. PHP.eB infusion into the cisterna magna of older adult rats compared to AAVI. Gene Ther. [in press].

Choudhury S.R., Hudry E., Maguire C.A., Sena-Esteves M., Breakefield X.O., Grandi, P. (2017) Viral vectors for therapy of neurologic diseases. Neuropharmacology 120: 63-80.

Dayton R.D., Grames M.S., Klein R.L. (2018) More expansive gene transfer to the rat CNS: AAV PHP.EB vector doseresponse and comparison to $A A V$ PHP.B. Gene Ther. 25(5): 392-400.

Düring D.N., Dittrich F., Rocha M.D., Tachibana R.O., Mori C., Okanoya K., Boehringer R., Ehret B., Grewe B.F., Gerber S. (2020) Fast retrograde access to projection neuron circuits underlying vocal learning in songbirds. Cell Rep. 33(6): 108364.

Hadaczek P., Eberling J.L., Pivirotto P., Bringas J., Forsayeth J., Bankiewicz K.S. (2010) Eight years of clinical improvement in MPTP-lesioned primates after gene therapy with AAV2-hAADC. Mol. Ther. 18: 1458-1461.

Haenraets K., Foster E., Johannssen H., Kandra V., Frezel N., Steffen T., Jaramillo V., Paterna J.C., Zeilhoter H.U., Wildner H. (2017) Spinal nociceptive circuit analysis with recombinant adeno associated viruses: the impact of serotypes and promoters. J. Neurochem. 142(5): 721-733.

Hashimoto M., Yamanaka A., Kato S., Tanifuji M., Kobayashi K., Yaginuma H. (2018) Anatomical evidence for a direct projection from Purkinje cells in the mouse cerebellar vermis to medial parabrachial nucleus. Front. Neural Circ. 12: 6.

Itoga C.A., Chen Y., Fateri C., Echeverry P.A., Lai J.M., Delgado J., Badhon S., Short A., Baram T.Z., Xu X. (2019) New viral genetic mapping uncovers an enrichment of corticotropin releasing hormone expressing neuronal inputs to the nucleus accumbens from stress related brain regions. J. Compar. Neurol. 527(15): 2474-2487.

Jara J.H., Villa S.R., Khan N.A., Bohn M.C., Özdinler P.H. (2012) AAV2 mediated retrograde transduction of corticospinal motor neurons reveals initial and selective apical dendrite degeneration in ALS. Neurobiol. Dis. 47(2): 174-183.

Kaspar B.K., Erickson D., Schaffer D., Hinh L., Gage F.H., Peterson D.A. (2002) Targeted retrograde gene delivery for neuronal protection. Mol. Ther. 5(1): 50-56.

Klaw M.C., Xu C., Tom V.J. (2013) Intraspinal AAV injections immediately rostral to a thoracic spinal cord injury site efficiently transduces neurons in spinal cord and brain. Mol. Ther. Nucl. Acids 2: e108.

Klein R.L., Dayton R.D., Tatom J.B., Henderson K.M., Henning P.P. (2008) AAV8, 9, Rh10, Rh43 vector gene transfer in the rat brain: effects of serotype, promoter and purification method. Mol. Ther. 16: 89-96.

Li H., Illenberger J.M., Cranston M.N., Mactutus C.F., McLaurin K.A., Harrod S.B., Booze R.M. (2019) Posterior ventral tegmental area-nucleus accumbens shell circuitry modulates response to novelty. PLoS One 14(3): $\mathrm{e} 0213088$.

Löw K., Aebischer P., Schneider B.L. (2013) Direct and retrograde transduction of nigral neurons with $A A V 6,8$, and 9 
and intraneuronal persistence of viral particles. Human Gene Ther. 24(6): 613-629.

Masamizu Y., Okada T., Kawasaki K., Ishibashi H., Yuasa S., Takeda S., Nakahara K. (2011) Local and retrograde gene transfer into primate neuronal pathways via adeno-associated virus serotype 8 and 9. Neuroscience 193: 249-258.

Nathwani A.C., Rosales C., McIntosh J., Rastegarlari G., Nathwani D., Raj D., Nawathe S., Waddington S.N., Bronson R., Jackson S., Donahue R.E. (2011) Long-term safety and efficacy following systemic administration of a self-complementary $A A V$ vector encoding human FIX pseudotyped with serotype 5 and 8 capsid proteins. Mol. Ther. 19: 876-885.

Passini M.A., Macauley S.L., Huff M.R., Taksir T.V., Bu J., Wu I.H., Piepenhangen P.A., Dodge J.C., Shihabuddin L.S., O'Riordan C.R. (2005) AAV vector-mediated correction of brain pathology in a mouse model of Niemann-Pick $A d i-$ sease. Mol. Ther. 11(5): 754-762.

Parfitt G.M., Nguyen R., Bang J.Y., Aqrabawi A.J., Tran M.M., Seo D.K., Richards B.A., Kim, J.C. (2017) Bidirectional control of anxiety-related behaviors in mice: role of inputs arising from the ventral hippocampus to the lateral septum and medial prefrontal cortex. Neuropsychopharmacology 42(8): 1715-1728.

Pietersz K.L., Martier R.M., Baatje M.S., Liefhebber J.M., Brouwers C.C., Pouw S.M., Fokkert L., Lubelski J., Petry A., Blits B. (2021) Transduction patterns in the CNS following various routes of $A A V-5$-mediated gene delivery. Gene Ther. 28: 435-446.

Piguet F., Sondhi D., Piraud M., Fouquet F., Hackett N.R., Ahouansou O., Vanier M.T., Bieche I., Aubourg P., Crystal R.G. (2012) Correction of brain oligodendrocytes by $A A V$ rh.10 intracerebral gene therapy in metachromatic leukodystrophy mice. Human Gene Ther. 23(8): 903-914.

Pina M.M., Cunningham C.L. (2017) Ethanol-seeking behavior is expressed directly through an extended amygdala to midbrain neural circuit. Neurobiol. Learn. Memory 137: 83-91.

Reardon T.R., Murray A.J., Turi G.F., Wirblich C., Croce K.R., Schnell M.J., Jessel T.M., Losonczy A. (2016) Rabies virus CVS-N2c $\Delta G$ strain enhances retrograde synaptic transfer and neuronal viability. Neuron 89(4): 711-724.

Taymans J.M., Vandenberghe L.H., Haute C.V.D., Thiry I., Deroose C.M., Mortelmans L., Wilson J.M., Debyser Z., Baekelandt V. (2007) Comparative analysis of adeno-associated viral vector serotypes $1,2,5,7$, and 8 in mouse brain. Human Gene Ther. 18(3): 195-206.

Tervo D.G.R., Hwang B.Y., Viswanathan S., Gaj T., Lavzin M., Ritola K.D., Lindo S., Michael S., Kuleshove E., Pjala D. (2016) A designer $A A V$ variant permits efficient retrograde access to projection neurons. Neuron 92(2): 372-382.

Wang J., Zhang L. (2021) Retrograde axonal transport property of adeno-associated virus and its possible application in future. Microbes Infec. 23(8): 104829.

Xie B.S., Wang X., Pan Y.H., Jiang G., Feng J.F., Lin Y. (2021) Apolipoprotein E, low-density lipoprotein receptor, and immune cells control blood-brain barrier penetration by AAV-PHP.eB in mice. Theranostics 11(3): 1177.

Zheng N., Wang Z.Z., Wang S.W., Yang F.J., Zhu X.T., Lu C., Manyande A., Rao X.P., Xu F.Q. (2020) Co-localization of two-color rAAV2-retro confirms the dispersion characteristics of efferent projections of mitral cells in mouse accessory olfactory bulb. Zoolog. Res. 41(2): 148. 